\title{
MAP17 and the double-edged sword of ROS
}

\section{Amancio Carnero}

Instituto de Biomedicina de Sevilla (IBIS), HUVR/CSIC/Universidad de Sevilla, Consejo Superior de Investigaciones Científicas, Spain

Corresponding author: Amancio Carnero

Instituto de Biomedicina de Sevilla - IBiS/HUVR

Consejo Superior de Investigaciones Cientificas

Campus Hospital Universitario Virgen del Rocío. Edificio IBIS

Avda. Manuel Siurot s/n

41013 Sevilla (Spain)

Phone: 955923111

Fax: 955923101

acarnero-ibis@us.es 


\section{$\underline{\text { ABSTRACT }}$}

Reactive oxygen species, ROS, are beneficially involved in many signaling pathways that control development and maintain cellular homeostasis. In physiological conditions, a tightly regulated redox balance protects cells from injurious ROS activity, but if the balance is altered, it promotes various pathological conditions including cancer. Understanding the duality of ROS as cytotoxic molecules and key mediators in signaling cascades may provide novel opportunities for improved cancer therapy.

MAP17 is a small 17-kDa non-glycosylated membrane protein that is overexpressed in many tumors of different origins, including carcinomas. Immunohistochemical analysis of MAP17 during cancer progression demonstrates that overexpression of the protein strongly correlates with the progression of most types of tumor. Tumor cells that overexpress MAP17 show an increased tumoral phenotype associated with an increase in ROS. However, in non-tumor cells MAP17 increases ROS, resulting in senescence or apoptosis. Therefore, in tumor cells, MAP17 could be a marker for increased oxidative stress and could define new therapeutic approaches. Here, we review the role of MAP17 as a putative oncogene, as well as its role in cancer and anticancer therapy.

Keywords: MAP17, Cancer, Oncogene, Reactive Oxygen Species, Tumorigenesis. 


\section{An Introduction to ROS}

ROS may promote either proliferation or cell death, depending on the intensity and location of the oxidative burst and the activity of the antioxidant system [1, 2]. Considering the proliferation signals delivered by ROS to cancer cells, and the consequent resistance of cancer cells to pro-apoptotic signals, ROSinduced tumor cell death is likely to be induced by ROS-generating antineoplastic therapies that increase the constitutive oxidative stress above the critical threshold required for cell death.

Cancer cells develop an enhanced constitutive oxidative stress that sustains tumor growth and protects these cells against proapoptotic signals, thus promoting tumor progression [1, 3]. In experimental models, it has been shown that ROS generation in tumors and the subsequent oxidative stress actually occur at sublethal levels [4]. However, many therapeutic drugs, as well as radiotherapy and photodynamic therapy, kill cancer cells, at least in part by increasing ROS [1, $5,6]$.

Evidence exists that the role of ROS in cancer is not limited to the generally accepted genotoxic and mutagenic effects that initiate cancer. As signal transduction messengers, ROS may promote the proliferation, senescence or death of cancer cells, depending on the actual intracellular and exogenous conditions. ROS have been shown to modulate growth signals and to activate expression leading to the sustained proliferation of cancer cells $[3,7]$. An emerging view is that upon oncogenic transformation, cells rapidly activate a stress response as a protective measure to overcome oncogene-induced cell death and senescence [8]. Cancer cells subject to persistent endogenous and exogenous oxidative stress were shown to develop adaptive responses primarily related to the upregulation of the antioxidant machinery. Therefore, cancer cells might become resistant to both enhanced constitutive stress and ROS-generating therapies, limiting the efficacy of the latter.

Current "ROS threshold" theories suggest that along with increases in ROS, cell responses change from proliferation to balance and then to cell death after ROS surpass a certain level [9]. Therefore, normal cells differ from tumor cells in their ability to control redox homeostasis. In normal cells, the use of antioxidants to scavenge free radicals protects from ROS-induced malignant transformation. In 
tumors, antioxidant or low levels of ROS that induce antioxidant defenses appear to benefit tumor growth and could enhance anticancer therapy resistance [9]. However, redox loading of tumor cells increases ROS to close to threshold levels. Then, when both normal and tumor cells are exposed to ROS-inducing therapies, the ROS levels in tumor cells will more easily reach levels that cause cell death [6]. This theory indicates that modest increases of ROS are oncogenic, while high ROS levels suppress tumors.

Accumulating evidence implicates ROS in signaling cascades related to cell proliferation and transformation [4, 10, 11]. Ras-transformed fibroblasts overproduce ROS, and this overproduction is correlated with the activation of mitogenic signaling pathways [10]. Loss of superoxide-dismutase, MnSOD, which should elevate ROS levels, has also been correlated with a tumoral phenotype, and overexpression of MnSOD leads to the reversion of the transformed phenotype [12-15]. On the other hand, $\mathrm{H}_{2} \mathrm{O}_{2}$ is generated in response to the growth factors EGF and PDGF and is linked to growth-related signaling [1 1, 16]. When overexpressed in NIH 3 T3 mouse fibroblasts, Nox1, an NADPH oxidase catalytic subunit, induces excessive production of ROS and a transformed phenotype with increased mitotic rates and aggressive tumor formation in athymic mice [17]. The phenotype of Noxl-transfected cells can be reversed by ROS reduction through stable expression of catalase, thereby implicating ROS as a signaling molecule [17]. Although later experiments do not support that claim [18, 19], high levels of Noxl with increased levels of ROS have been detected in colon and prostate samples [18-22]. Nox4 was shown to sustain prostate cancer cell survival after activation by signals provided by the extracellular matrix [23]. On the other hand, decreased activity of antioxidants has been observed in ovarian cancer patients [24]. Decreased expression and activity of mitochondrial MnSOD have been reported in colorectal and pancreatic carcinomas correlated with altered redox status $[25,26]$. Furthermore, various oncogenic signals such as those induced by oncogenic Raf, c-myc or Bcr-Abl were shown to be involved in increasing ROS generation via the NADPH oxidase pathway $[27,28]$.

The cellular targets responsible for growth and transformation affected by ROS signaling are not well known. The p42/p44 mitogen-activated protein kinase (MAPK), p38 MAPK, p70S6k, signal transducers and activators of transcription 
(STAT), Akt/Protein Kinase B and phospholipase D signaling pathways are all activated by reactive oxygen species [16, 29-31]; however, in some cases, activation is indirect $[32,33]$. A direct effect has been shown for protein tyrosine phosphatase-1B (PTP-1B), which is inhibited by oxidation of a thiol in the active site $[34,35]$ leading to increased phosphotyrosines on many cell proteins. Furthermore, ROS increase activates the PI3K pathway by direct oxidation and inactivation of PTEN and other AKT phosphatases, thus maintaining AKT activation even in the absence of a PI3K signal [36]. A variety of other targets can also be affected by ROS, including transcription factors such as NF-kB [37], activator protein-1 (AP1) [38], PTEN [39] and p53 [40]. ROS can directly modify signaling proteins through different modifications such as nitrosylation, carbonylation, disulfide bond formation and glutathionylation [41]. Whatever the proximal target(s), ROS can reprogram the expression of enzymes and other proteins in the cell $[42,43]$. DNA microarray experiments [17] indicate that up to $2 \%$ of the genes are transcriptionally regulated by ROS.

\section{MAP17 is overexpressed in cancer}

Functional genetic screens using retroviral delivery of high complexity cDNA libraries are valuable tools to discover new genes involved in a particular phenotypic characteristic of the tumorigenic process [44-46]. A genome-wide retroviral cDNA screen to search for genes that confer a selective advantage to cancer cells during tumorigenesis allowed us to identify MAP17 [47]. MAP17 is a small, non-glycosylated, membrane-associated 17-kDa protein that localizes to the plasma membrane and the Golgi apparatus [48]. The protein sequence contains two transmembrane regions and a hydrophobic amino-terminus of 13 amino acids encoding a PDZ-binding domain [49]. MAP17 overexpression in carcinomas was first detected by using the technique of differential display [50]. Transfection of full-length wild-type MAP17 into HT29 colon carcinoma cells decreased cell proliferation in vitro and tumor growth in vivo [51]. MAP17 binds several PDZ domain-containing proteins, including PDZK 1, NHERF1, NaPi-lla and NHe3. Overexpression of MAP17 in opossum kidney cells participates, together with PDZK 1 and NH3RF4, in NaPi-lla internalization to the trans-Golgi network [52]. The physiological role of MAP17 in proximal tubules is not well known, but it does stimulate specific Na-dependent transport of mannose and glucose in Xenopus 
oocytes [48] and human tumor cells [47]. The MAP17 gene shares regulatory elements with the stem cell leukemia gene (SCL, TAL-1), which encodes a basic Helix-Loop-Helix protein essential in the formation of the hematopoietic lineages $[53,54]$. However, in non-cancerous tissues, major expression of MAP17 has only been detected in kidney cells.

In a meta-analysis of public microarray databases for different skin diseases, Noh et al. [55] discovered that MAP17 is commonly upregulated, suggesting that it may be associated with abnormal keratinocyte differentiation. MAP17 was significantly upregulated in response to interferon-gamma, interleukin 4 (IL-4), IL-6, IL-17A or IL-22 in normal human epidermal keratinocytes. Interestingly, the PDZK I gene is localized within the atopic dermatitis-linked region on human chromosome 1q21. In an attempt to evaluate whether MAP17 regulates the expression of cornified envelope-associated genes at the 1q21 locus, such as filaggrin, loricrin and involucrin, these authors [55] found that the over-expression of MAP17 in HaCaT keratinocytes significantly decreased the expression of filaggrin, a cornified envelope-associated gene. Taken together, the Th cell cytokine-induced upregulation of MAP17 expression may be linked to the downregulation of filaggrin, which may be associated with the abnormal epidermal differentiation observed in the dermatological diseases [55].

Human MAP17 maps to chromosome 1p33, a locus commonly found to be involved in cancer; however, it is not the only interesting gene in this region. Genes coding for members of the cytochrome P450 family (CYP4B1, CYP4A1 1), putative oncogenes (SCL/Tal1), MCPH7, CMPK1, and members of the forkhead family (FOXE3, FOXD2) are its neighbors.

MAP17 overexpression has previously been shown to be associated with carcinomas [50, 51]. An in-depth analysis of MAP17 overexpression in carcinomas by immunohistochemistry and mRNA expression showed that the MAP17 protein is overexpressed in a large percentage of the tumors analyzed (Figure 1) and is significantly correlated with the tumor grade at least in ovarian, breast and prostate carcinomas [56]. A comparison of tumoral with non-tumoral tissues of the same patient by hybridization or by analyzing mRNA levels by Q-PCR 
demonstrated an even higher percentage of tumor samples with MAP17 overexpression [56]. Overexpression was observed in more than $70 \%$ of the samples from tumors such as ovary, colon, stomach, cervix and thyroid and in approximately $50 \%$ of the samples from tumors of the lung, uterus and rectum. Although more samples need to be analyzed to confirm these high frequencies, the data suggest that MAP17 overexpression is the most common marker of tumorigenesis in carcinomas. The relevance of MAP17 as a general marker for the malignant stages of human tumors still needs to be confirmed in additional tumor types and larger cohorts. Furthermore, MAP17 expression seems to correlate with AKT473 phosphorylation and p38 T180/Y182 dephosphorylation [57].

MAP17 overexpression in carcinomas occurs mostly through mRNA amplification (Figure 2). MAP17 overexpression could be due to the ability of the MAP17 promoter to be activated by oncogenes $[50,56]$. Tumorigenic progression involves progressive genetic alterations triggering oncogenic cascades [58]. In advanced stages, tumors might accumulate oncogenic alterations that result in a high probability of MAP17 promoter activation and increased transcription. This hypothesis could explain the correlation between the MAP17 overexpression and advanced tumor stages observed in many tumor types.

However, the preceding data do not provide an explanation of why MAP17 overexpression provides a selective advantage during tumorigenesis. Multiple oncogenes that activate signaling pathways directly involved in cell survival or proliferation have been discovered in previous decades. Other genes may provide an advantage to the tumoral cells, making them insensitive to physiological signals or altering their normal physiology. Although activated macrophages destroy cancer cells more effectively than normal cells, the ability to escape activated macrophages is a characteristic of tumor cells. One of the mechanisms responsible for the specific killing of tumor cells by macrophages is the production of the cytokine tumor necrosis factor alpha (TNF). Therefore, resistance to TNF may provide cancer cells with a selective advantage against host elimination. Ectopic expression of MAP17 in tumor cells prevents TNF-induced G1 arrest by impairing p21wafl induction. However, expression of MAP17 does not inhibit TNF-induced apoptosis in Me180-sensitive tumor cells. The inhibition of TNF is 
specific because MAP17 does not alter the response to other cytokines, such as IFNa. As described in the Xenopus oocyte system, MAP17 increases the uptake of mannose in some cells, but this effect is not responsible for TNF bypass [47].

\section{MAP17 expression enhances the tumorigenic phenotype by increasing intracellular ROS}

Tumor cells that overexpress MAP17 show an increased tumoral phenotype with enhanced proliferative capabilities, both in the presence and absence of contact inhibition, decreased apoptotic sensitivity and increased migration [59]. MAP17-expressing clones also grow better in nude mice. The increased malignant cell behavior induced by MAP17 is associated with an increase in ROS production, and the treatment of MAP17-expressing cells with antioxidants results in a reduction in the tumorigenic properties of these cells. The MAP17-dependent increase in ROS and tumorigenesis is dependent on its PDZ-binding domain because disruption of this sequence by point mutations abolishes the ability of MAP17 to enhance ROS production and tumorigenesis $[57,59]$.

MAP17 also decreases the c-Myc-induced caspase-3-like activity in Rat1 fibroblasts under low serum conditions. This decrease is in keeping with the concept of MAP17-induced PI3K/AKT signaling, in which MAP17 is able to interfere with Bax translocation to the mitochondria [36]. A fraction of PTEN protein is oxidized in MAP17-overexpressing cells. Furthermore, activation of AKT by MAP17 as measured by Thr308 phosphorylation is independent of PI3K activity. Importantly, modulation of ROS by antioxidant treatment prevented activation of AKT, thus restoring the level of apoptosis in serum starved Ratl/c-Myc fibroblasts [36]. Therefore, overexpression of MAP17 protects Ratla fibroblasts from Mycinduced apoptosis through ROS-mediated activation of the PI3K/AKT signaling pathway [36].

The increased tumorigenic properties induced by MAP17 are associated with an increase in ROS because MAP17 increases endogenous ROS, and antioxidant treatment of MAP17-expressing cells entails a reduction in the tumorigenic properties of these cells. Two explanations can be offered for the mechanism by which ROS induce the transformed phenotype. First, reactive 
oxygen generated in the presence of MAP17 may be mutagenic, causing the transformed phenotype through the induction of mutations in oncogenes or tumor suppressor genes. Alternatively, ROS generated in a MAP17-dependent manner might function as an intracellular signal, inducing a growth-related genetic program. ROS removal by antioxidant treatments decreases the malignant cell behavior induced by MAP17; thus, the second hypothesis is favored. Furthermore, in breast carcinoma cells, elimination of ectopically expressed MAP17 reduced the tumorigenic capabilities [57]. This reversibility of the phenotype indicates that the effect induced by MAP17 is largely independent of ROS-induced DNAmutations.

However, the increased tumoral properties of carcinoma cells were not paralleled in naïve non-tumoral cells [57], indicating that MAP17 provides a selective advantage once tumorigenesis has begun. Our data demonstrate that ROS act as a second messenger that enhances tumoral properties but only in those cells where the senescence/apoptotic signal provided by ROS is uncoupled. We have found that p38a activation at least partly mediates this response. MAP17 triggers a ROS-dependent, senescence-like response that is abolished in the absence of p38a activation. Furthermore, in human breast tumors, MAP17 activation is correlated with lack of p38a phosphorylation. Therefore, MAP17 is overexpressed in late-stage breast tumors, in which oncogenic activity relies on p38 insensitivity to induced intracellular ROS [57].

\section{ROS increase}

How are ROS increased by MAP17? One possibility is that MAP17 can increase glucose and mannose uptake, inducing an increase in metabolism with ROS as side products. However, it is also possible that a direct link with the membrane transporters ends, altering the intracellular redox balance by altering the intra/extracellular ion balance.

The structural simplicity of MAP17 and the kinetic analysis of the induced membrane transport [60] suggest that MAP17 is an activator of the capacity of endogenous uphill transporters. MAP17 could modulate the activity or the organization of membrane transporters through direct interaction as the RS1 
modifier does [61] or through competition for PDZ-binding domains to alter the stoichiometry of the transporter-PDZ proteins [60].

\subsection{MAP17 binding to PDZK1 (NHeRF3, CAP70, NaPi-Capl and CLAM)}

Kocher et al. [62] first described the interaction between MAP17 and PDZK I detected by using a two-hybrid system. In a transgenic mouse model, MAP17 hepatic overexpression resulted in a liver deficiency of PDZK 1, suggesting that MAP17 is an endogenous regulator of PDZK 1 turnover [63]. MAP17 acts as an atypical anchoring site for PDZK1 and interacts with the NaPi-lla/PDZK1 protein complex in renal proximal tubular cells [64].

PDZK 1 belongs to the NHeRF (sodium hydrogen exchange regulatory factor) family, members of which are PDZ domain-containing proteins that play important roles in regulating cell function [65]. This family contains four members, NHeRF1, NHeRF2, PDZK1 and IKEPP (NHeRF4), that share similar homology domains [65]. NHeRFland NHeRF2 each contain 2 PDZ domains, while PDZK 1 and IKEPP each contain 4 PDZ domains [66]. NHeRF1 was initially identified as a membraneassociated protein that is essential for the regulation of the PKA-induced inhibition of the $\mathrm{Na}-\mathrm{H}$ exchange isoform 3 (NHe3). NHeRF2 was also identified as a mediator of NHe3 inhibition by cAMP. Although the different members of the family carry some overlapping functions, it is clear that there is a significant amount of specificity among them [67]. ThUs, PDZK 1 is a typical scaffolding protein defined by the presence of globular PDZ domains that assemble several proteins into functional complexes. PDZK 1 is a critical spatio-temporal regulator of intracellular signaling in response to specific stimuli [68]. MAP17 has been shown to interact with PDZK 1 and NHeRF1 [69] (Figure 3A).

The NHeRF proteins are primarily expressed in the polarized epithelial cells at the apical side. Kidney, small intestine and liver tissues exhibit the highest expression levels of these proteins $[70,71]$.

The NHeRF proteins regulate cell surface expression and functional activity of transporters. Most transporters identified as binding partners belong to the $A B C$ family [66]. In addition to transporters, other proteins have been shown to interact with NHeRF proteins, including signaling proteins, hormone receptors and cytoskeleton structural elements $[65,72]$. Many proteins related to the G-protein 
signaling pathways were found to interact with PDZK 1, and they were likely to be functionally associated with transporters.

PDZK 1 forms heterooligomers with NHeRF1 in vitro, which may allow the formation of an entire network of PDZ adapter proteins underneath the plasma membrane [73].

MAP1 7 complexes with PDZK 1 and NHe3 contributing to basal and calcium inhibition of NH3 activity [74]. PDZK1 also regulates the solute carriers SLC15al (oligopeptide transporter, PEPT1) and SLC22a5 (carnitine/organic cation transporter, OCTN2) in the small intestine [75], the cystic fibrosis transmembrane conductance regulator (CFTR) and the anion exchangers of the SLC26A family, leading to its stabilization [76]. PDZK 1 has also been shown to interact with AKAP10, FARP2, sodium-hydrogen antiporter 3 regulator 1, SLC22A12, SLK, SLC22A4 [77], CLCN3, cystic fibrosis transmembrane conductance regulator [78] and SLC34A3 [77]. Coupling CFTR to DRA and PATl results in activation of the $\mathrm{Cl} / / \mathrm{HCO}_{3}-$ exchange. As a consequence of transport regulation, binding of MAPI7 to NHeRF proteins might result in the deregulation of the intracellular and extracellular cation/anion balance, causing increased nonenzymatic oxidative stress in the cells.

On the other hand, PDZK 1 interacts with CMOAT, a canalicular multispecific organic anion transporter involved in multidrug resistance. This finding was considered of particular interest because proteins containing PDZ domains are involved in the clustering and signaling pathways of membrane-associated proteins, including ion channels. Therefore, the protein cluster formed by the association of CMOAT, PDZK1, and MAP17 could play an important role in the cellular mechanisms associated with multidrug resistance, and PDZK I may represent a new target in cancer cells resistant to chemotherapeutic agents. In line with this argument, the overexpression of PDZK 1 within the 1q12-q22 amplicon is likely to be associated with the drug resistance phenotype in multiple myeloma [79].

\subsection{SGLTs and Gluts transporters}

The Na-dependent glucose transporter 1 (SGLT1, SCL5A1) is the main mediator of apical glucose uptake, whereas at the basolateral membrane the glucose transporter GLUT1 facilitates diffusive transport of intracellular glucose into 
the bloodstream [80]. SGLT1 mRNA is present mainly in the intestine and kidney [81, 82]. Located at 22q13.1, SGLT1 is responsible for active glucose absorption, which is an energy requiring action driven by the $\mathrm{Na}+\mathrm{K}+$ ATPase [83].

MAP17 binds and activates specific Na-dependent transport of mannose and glucose in Xenopus oocytes [48]. The induced transport has the functional characteristics of the Na-glucose cotransporters, the SGLTs [60]. Ectopic expression of MAP17 in human cells, tumoral or not, triggers increased absorption of glucose and mannose [47]. This transport is inhibited by phloridzin, suggesting that the increase is due to the SGLT1 transporter, corroborating the previous finding in Xenopus [47]. We have also found that MAP17 and SGLT1 colocalize in some tumors (Data not shown).

Previous studies demonstrated that activation of SGLT1 rescued enterocytes from apoptosis by activating PI3K [84] and that inhibition of this membrane transport with phloridzin also inhibited MAP17-dependent ROS increase and proliferation [59]. Together, these results suggest that MAP17-dependent tumorigenic properties may depend upon the activation of ROS by SGLT1 membrane transport. SGLT1, on the other hand, has been previously related to cancer [85], showing correlation with prognosis. Furthermore, silencing EGFR decreases SGLTI significantly reducing the intracellular concentrations of glucose [86]. Despite what seems a clear functional relationship, no direct evidence for binding of MAP17 to SGLT1 in mammalian cells has been published.

In normal cells under aerobic conditions, the oxidation of glucose involves cytoplasmic glycolysis and oxidative phosphorylation by the mitochondrial electron transport chain, which produces a maximum of ATP by completely oxydizing glucose to $\mathrm{CO}_{2}$. Under hypoxic conditions, normal cells perform anaerobic glycolysis because mitochondrial function is suppressed in the absence of $\mathrm{O}_{2}$. Anaerobic glycolysis generates lactate from pyruvate, which is the only way to regenerate NAD+, the coenzyme for glyceraldehyde 3-phosphate dehydrogenase. Despite the low energy produced in anaerobic glycolysis (16 fold lower than oxidative phosphorylation), tumor cells largely rely on the conversion of glucose to lactate rather than on mitochondrial oxidation for energy production 
even in the presence of high oxygen levels [87-91]. This effect is known as the Warburg effect, or aerobic glycolysis $[92,93]$.

Tumor cells, therefore, have mostly inhibited oxidative phosphorylation, reducing ATP production. They do not allow electrons to go through the electron transport chain all the way to oxygen, thus increasing the generation of ROS [9395].

Thus, reduced ATP generation in mitochondria is a compromise that tumor cells make to initiate oncogenic transformation. Tumor cells must increase their access to glucose to support the high rate of glycolysis. This is achieved by an increased transport of glucose into the cells. The rate of glucose entry into tumor cells is at least 20- to 30-fold higher than in normal cells [91]. Tumor cells enhance glucose uptake via the induction of Glutl and SGLT1, and coordinate the increased entry of glucose with increased glycolysis $[91,96,97]$.

Pyruvate, but not lactate, is an effective inhibitor of histone deacetylases $[98,99]$. Therefore, accumulation of pyruvate in tumor cells can potentially kill them, and aerobic glycolysis must be maintained to eliminate pyruvate and produce the lactate that will be used for essential NAD+ production.

On the other hand, an increased level of lactate in the cytoplasm will decrease cellular $\mathrm{pH}$, potentially compromising survival. Tumor cells employ many mechanisms to prevent cellular acidification [100]. Cellular acidification results from enhanced glucose uptake and anaerobic metabolism by tumor cells. This acidic intracellular $\mathrm{pH}$ can be exacerbated by inefficient removal of lactic acid, $\mathrm{CO} 2$ and $\mathrm{H}+$ by deficient vasculature in the tumor [101]. Since small variations in the intracellular $\mathrm{pH}$ alter many biological functions such as membrane permeability, enzymatic activity, ATP maintenance and production, proliferation, migration, invasion, metastasis or drug resistance and apoptosis, the cells must regulate the intracellular $\mathrm{pH}$ to survive. The hypoxia inducible factor, HIFl, regulates under hypoxic conditions anaerobic glycolysis and $\mathrm{pH}$ homeostasis by enhancing both, the expression of glycolytic enzymes and the membrane located transporters, exchangers and ecto-enzymes [102]. The system regulating the intracellular $\mathrm{pH}$ in tumor cells actively export acids via the NHel exchanger [103105], the monocarboxilate transporters (MCTs) [106], and, to induce cytoplasmatic alkalinization, transports $\mathrm{HCO} 3$ - into the cells through $\mathrm{Cl}-/ \mathrm{HCO} 3$ - exchangers and 
$\mathrm{Na}+\mathrm{HCO} 3-$ cotransports [107]. The $\mathrm{NHel}$ is also enhanced in many tumors pumping $\mathrm{H}+$ out of the cells coupled to a transmembrane gradient. Additionally, $\mathrm{H}+$ /lactate-coupled transporters, carbonic anhydrases, could facilitate $\mathrm{H}+$ efflux, preventing acidification of the cytoplasm [108].

Most of these membrane transporters could be regulated by MAP17 levels and localization through NHeRF proteins binding (Figure 3B). Therefore, the MAP17/NHeRF complexes could be an intracellular pH/acidosis detoxifying system parallel to carbonic anhydrases. It is possible that MAP17/NHeRFs-dependent system be also induced under hypoxic conditions maintained through further malignization. However, the fact that levels of MAP17 rise with the stage of the tumor suggests that may be more related to continuous selection of cells with higher $\mathrm{pH}$ detox capabilities. Further experimental validation is necessary to clarify these hypotheses.

By activating MAP17, cells will insure increased membrane localization of internal $\mathrm{pH}$ detoxifying transporters. Several oncogenes could activate MAP17 expression [56] promoting MAP17 increase along with increased metabolism. Unspecific membrane transporter inhibitors, such as furosemide, produce similar effects on cellular proliferation than phloridzin, an SGLT inhibitor, suggesting the functional relationship between SGLT and the $\mathrm{pH}$ regulation [59]. Thus, enhanced membrane transport to prevent acidification may be regulated by MAP17 through NHeRF binding allowing SGLT1 increase and anerobic glycolysis activation in tumor cells.

However, it is also possible that secondary effects of an increase in glucose and mannose, such as glycosylation and mannosylation of proteins might alter properties of the cells, including oxidative stress or tumorigenic properties, thus contributing to the MAP17-induced tumoral phenotype.

\section{MAP17 and cancer therapy}

Treatment of melanoma cells with inhibitors of $\mathrm{Na}^{+}$-coupled cotransporters leads to an inhibition of a ROS increase and a decrease in the malignant cell behavior in MAP17-expressing clones [59]. These changes could result in a new therapeutic approach by nonspecifically blocking uphill transport in tumor cells. 
However, the rapid adaptation of the cells to these pharmacologic interventions and possible toxic effects need to be better studied.

A low level of ROS is indispensable in several physiological processes of the cell, including proliferation and apoptosis, or cell death [109]. A mild increase in ROS has been shown to activate signaling cascades which can seriously influence the regulation of cell growth and tumorigenic processes $[4,10,11,16,31,42,43$, 57, 110]. However, a further increase in ROS levels raises oxidative stress and creates a potentially toxic environment for the cell. In normal physiological conditions, a balance between ROS generation and oxidative defenses exists in the cell. In these defenses, endogenous antioxidant enzymes play a significant role. Superoxide dismutase (SOD) and catalase (CAT) that act on $\mathrm{O}_{2}$ - and $\mathrm{H}_{2} \mathrm{O}_{2}$, respectively, glutarredoxins, glutathione peroxidases (GPXs) that use glutathione as a cosubstrate, peroxiredoxins and thioredoxins, are in a delicate balance with oxidative inputs $[1,110]$. Although many cells can tolerate limited doses of ROS, when the balance tips further in favor of ROS, programmed cell death becomes a certainty [ $\left[\begin{array}{lll}1 & 1\end{array}\right]$. Cellular detoxification enzymes cannot neutralize excessive ROS that alter the chemical environment in the cells, especially within the mitochondria, and launch the cell death program.

Therefore, we can hypothesize that tumors expressing high levels of MAP17 and producing ROS can benefit from therapies that increase oxidative stress, such as cisplatin and radiotherapy, doxorubicin or camptothecin, which have redoxmediated activity [112] on tumor cells without affecting healthy tissues [113]. Tumors with high MAP17 can also benefit from combined therapies using cytotoxic agents and increased oxidative stress. The first attempt to employ pro-oxidant agents in vivo was reported by Nathan and Cohn in 1981. Using glucose oxidase as an $\mathrm{H}_{2} \mathrm{O}_{2}$ precursor, they obtained a significant decrease of tumor growth [114, $115]$.

Because cells can develop adaptive responses to ROS, primarily based on the increase of detoxifying enzymes [8], we can hypothesize that the inhibition of classical oxidative stress detoxifying enzymes can also increase the efficacy of certain antitumor therapies that increase ROS, at least in tumors that overexpress MAP17. However, the delicate balance between oxidative stress, cancer and cell 
death make it necessary to devise new experimental tests and develop a more complete understanding of these processes.

\section{CONCLUSIONS}

In summary, the observation that MAPI7 is overexpressed in human carcinomas indicates that MAP17 can be a good marker for tumorigenesis and for malignant progression. The results indicate that this protein is likely to play an important role in carcinogenesis and likely in the response to certain therapies related to oxidative stress. On the other hand, the overexpression of PDZK I within the 1q12-q22 amplicon is likely to be associated with the drug resistance phenotype in multiple myeloma [79]. All of these data suggest that the uphill transport and SGLT1-dependent glucose uptake might be important players in the correct balance of ROS in cells, indirectly regulating the ability to select more aggressive tumor cell populations. Therefore, a deeper understanding of this system might be of interest to the cancer field.

\section{ACKNOWLEDGEMENTS}

This work was supported by grants from the Spanish Ministry of Science and Innovation and Feder Funds (SAF2009-08605), Consejeria de Innovacion y Ciencia (CTS-6844) and Consejeria de Salud (PI-0142), Junta de Andalucia. AC's Lab is also funded by a fellowship from Fundacion Oncologica FERO supported by Fundació Josep Botet. The funding source has no involvement in the study.

\section{REFERENCES}

[1] G. Manda, Nechifor, M.T., Neagu, T.M., Reactive Oxygen species, cancer and anticancer therapies, Curr Chem Biol. 3 (2009) 342-366.

[2] R.H. Burdon, V. Gill, C. Rice-Evans, Oxidative stress and tumour cell proliferation, Free Radic Res Commun. 11 (1990) 65-76.

[3] L. Behrend, G. Henderson, R.M. Zwacka, Reactive oxygen species in oncogenic transformation, Biochem Soc Trans. 31 (2003) 1441-1444.

[4] R.H. Burdon, Control of cell proliferation by reactive oxygen species, Biochem Soc Trans. 24 (1996) 1028-1032.

[5] Y. Ge, J.S. Byun, P. De Luca, G. Gueron, I.M. Yabe, S.G. Sadiq-Ali, W.D. Figg, J. Quintero, C.M. Haggerty, Q.Q. Li et al, Combinatorial antileukemic disruption of oxidative homeostasis and mitochondrial stability by the redox reactive 
thalidomide 2-(2,4-difluoro-phenyl)-4,5,6,7-tetrafluoro-1H-isoindole-1,3(2H)-dione (CPS49) and flavopiridol, Mol Pharmacol. 74 (2008) 872-883.

[6] J. Wang, J. Yi, Cancer cell killing via ROS: to increase or decrease, that is the question, Cancer Biol Ther. 7 (2008) 1875-1884.

[7] W.C. Barrett, J.P. DeGnore, Y.F. Keng, Z.Y. Zhang, M.B. Yim, P.B. Chock, Roles of superoxide radical anion in signal transduction mediated by reversible regulation of protein-tyrosine phosphatase 1B, J Biol Chem. 274 (1999) 3454334546.

[8] M. Benhar, D. Engelberg, A. Levitzki, ROS, stress-activated kinases and stress signaling in cancer, EMBO Rep. 3 (2002) 420-425.

[9] Q. Kong, J.A. Beel, K.O. Lillehei, A threshold concept for cancer therapy, Med Hypotheses. 55 (2000) 29-35.

[10] K. Irani, Y. Xia, J.L. Zweier, S.J. Sollott, C.J. Der, E.R. Fearon, M. Sundaresan, T. Finkel, P.J. Goldschmidt-Clermont, Mitogenic signaling mediated by oxidants in Rastransformed fibroblasts, Science. 275 (1997) 1649-1652.

[11] M. Sundaresan, Z.X. YU, V.J. Ferrans, K. Irani, T. Finkel, Requirement for generation of $\mathrm{H} 2 \mathrm{O} 2$ for platelet-derived growth factor signal transduction, Science. 270 (1995) 296-299.

[12] T. Yan, L.W. Oberley, W. Zhong, D.K. St Clair, Manganese-containing superoxide dismutase overexpression causes phenotypic reversion in SV40-transformed human lung fibroblasts, Cancer Res. 56 (1996) 2864-2871.

[13] S.L. Church, J.W. Grant, L.A. Ridnour, L.W. Oberley, P.E. Swanson, P.S. Meltzer, J.M. Trent, Increased manganese superoxide dismutase expression suppresses the malignant phenotype of human melanoma cells, Proc Natl Acad Sci U S A. 90 (1993) 3113-3117.

[14] J.A. Fernandez-Pol, P.D. Hamilton, D.J. Klos, Correlation between the loss of the transformed phenotype and an increase in superoxide dismutase activity in a revertant subclone of sarcoma virus-infected mammalian cells, Cancer Res. 42 (1982) 609-617.

[15] J.L. Martindale, N.J. Holbrook, Cellular response to oxidative stress: signaling for suicide and survival, J Cell Physiol. 192 (2002) 1-15.

[16] G.U. Bae, D.W. Seo, H.K. Kwon, H.Y. Lee, S. Hong, Z.W. Lee, K.S. Ha, H.W. Lee, J.W. Han, Hydrogen peroxide activates p70(S6k) signaling pathway, J Biol Chem. 274 (1999) 32596-32602.

[17] R.S. Arnold, J. Shi, E. Murad, A.M. Whalen, C.Q. Sun, R. Polavarapu, S. Parthasarathy, J.A. Petros, J.D. Lambeth, Hydrogen peroxide mediates the cell growth and transformation caused by the mitogenic oxidase Noxl, Proc Natl Acad Sci U S A. 98 (2001) 5550-5555.

[18] M. Geiszt, K. Lekstrom, S. Brenner, S.M. Hewitt, R. Dana, H.L. Malech, T.L. Leto, NAD(P)H oxidase 1, a product of differentiated colon epithelial cells, can partially replace glycoprotein 91 phox in the regulated production of superoxide by phagocytes, J Immunol. 171 (2003) 299-306.

[19] M. Geiszt, K. Lekstrom, J. Witta, T.L. Leto, Proteins homologous to p47phox and p67phox support superoxide production by NAD(P)H oxidase 1 in colon epithelial cells, J Biol Chem. 278 (2003) 20006-20012.

[20] M. Fukuyama, K. Rokutan, T. Sano, H. Miyake, M. Shimada, S. Tashiro, Overexpression of a novel superoxide-producing enzyme, NADPH oxidase 1, in adenoma and well differentiated adenocarcinoma of the human colon, Cancer Lett. 221 (2005) 97-104.

[21] I. Szanto, L. Rubbia-Brandt, P. Kiss, K. Steger, B. Banfi, E. Kovari, F. Herrmann, A. Hadengue, K.H. Krause, Expression of NOX1, a superoxide-generating NADPH oxidase, in colon cancer and inflammatory bowel disease, J Pathol. 207 (2005) 164-176.

[22] S.D. Lim, C. Sun, J.D. Lambeth, F. Marshall, M. Amin, L. Chung, J.A. Petros, R.S. Arnold, Increased Noxl and hydrogen peroxide in prostate cancer, Prostate. 62 (2005) 200-207. 
[23] M. Edderkaoui, P. Hong, E.C. Vaquero, J.K. Lee, L. Fischer, H. Friess, M.W. Buchler, M.M. Lerch, S.J. Pandol, A.S. Gukovskaya, Extracellular matrix stimulates reactive oxygen species production and increases pancreatic cancer cell survival through 5-lipoxygenase and NADPH oxidase, Am J Physiol Gastrointest Liver Physiol. 289 (2005) G1137-1147.

[24] K. Senthil, S. Aranganathan, N. Nalini, Evidence of oxidative stress in the circulation of ovarian cancer patients, Clin Chim Acta. 339 (2004) 27-32.

[25] B.E. Van Driel, H. Lyon, D.C. Hoogenraad, S. Anten, U. Hansen, C.J. Van Noorden, Expression of CuZn- and Mn-superoxide dismutase in human colorectal neoplasms, Free Radic Biol Med. 23 (1997) 435-444.

[26] J.J. Cullen, F.A. Mitros, L.W. Oberley, Expression of antioxidant enzymes in diseases of the human pancreas: another link between chronic pancreatitis and pancreatic cancer, Pancreas. 26 (2003) 23-27.

[27] R.H. Xu, H. Pelicano, Y. Zhou, J.S. Carew, L. Feng, K.N. Bhalla, M.J. Keating, P. Huang, Inhibition of glycolysis in cancer cells: a novel strategy to overcome drug resistance associated with mitochondrial respiratory defect and hypoxia, Cancer Res. 65 (2005) 613-621.

[28] H. Pelicano, D. Carney, P. Huang, ROS stress in cancer cells and therapeutic implications, Drug Resist Updat. 7 (2004) 97-110.

[29] V. Natarajan, M.M. Taher, B. Roehm, N.L. Parinandi, H.H. Schmid, Z. Kiss, J.G. Garcia, Activation of endothelial cell phospholipase D by hydrogen peroxide and fatty acid hydroperoxide, J Biol Chem. 268 (1993) 930-937.

[30] R.G. Allen, M. Tresini, Oxidative stress and gene regulation, Free Radic Biol Med. 28 (2000) 463-499.

[31] T. Finkel, Oxygen radicals and signaling, Curr Opin Cell Biol. 10 (1998) 248-253.

[32] J. Abe, M. Okuda, Q. Huang, M. Yoshizumi, B.C. Berk, Reactive oxygen species activate p90 ribosomal S6 kinase via Fyn and Ras, J Biol Chem. 275 (2000) 17391748.

[33] D.S. Min, E.G. Kim, J.H. Exton, Involvement of tyrosine phosphorylation and protein kinase $\mathrm{C}$ in the activation of phospholipase D by H2O2 in Swiss 3T3 fibroblasts, J Biol Chem. 273 (1998) 29986-29994.

[34] S.R. Lee, K.S. Kwon, S.R. Kim, S.G. Rhee, Reversible inactivation of protein-tyrosine phosphatase 1B in A431 cells stimulated with epidermal growth factor, J Biol Chem. 273 (1998) 15366-15372.

[35] W.C. Barrett, J.P. DeGnore, S. Konig, H.M. Fales, Y.F. Keng, Z.Y. Zhang, M.B. Yim, P.B. Chock, Regulation of PTP1B via glutathionylation of the active site cysteine 215, Biochemistry. 38 (1999) 6699-6705.

[36] M.V. Guijarro, W. Link, A. Rosado, J.F. Leal, A. Carnero, MAP17 inhibits Myc-induced apoptosis through PI3K/AKT pathway activation, Carcinogenesis. 28 (2007) 24432450.

[37] K.N. Schmidt, P. Amstad, P. Cerutti, P.A. Baeverle, The roles of hydrogen peroxide and superoxide as messengers in the activation of transcription factor NF-kappa B, Chem Biol. 2 (1995) 13-22.

[38] J. Wenk, P. Brenneisen, M. Wlaschek, A. Poswig, K. Briviba, T.D. Oberley, K. Scharffetter-Kochanek, Stable overexpression of manganese superoxide dismutase in mitochondria identifies hydrogen peroxide as a major oxidant in the AP-1-mediated induction of matrix-degrading metalloprotease-1, J Biol Chem. 274 (1999) 25869-25876.

[39] S.R. Lee, K.S. Yang, J. Kwon, C. Lee, W. Jeong, S.G. Rhee, Reversible inactivation of the tumor suppressor PTEN by H2O2, J Biol Chem. 277 (2002) 20336-20342.

[40] P. Hainaut, J. Milner, Redox modulation of p53 conformation and sequencespecific DNA binding in vitro, Cancer Res. 53 (1993) 4469-4473.

[41] K. England, T.G. Cotter, Direct oxidative modifications of signalling proteins in mammalian cells and their effects on apoptosis, Redox Rep. 10 (2005) 237-245.

[42] J.E. Klaunig, Y. XU, J.S. Isenberg, S. Bachowski, K.L. Kolaja, J. Jiang, D.E. Stevenson, E.F. Walborg, Jr., The role of oxidative stress in chemical carcinogenesis, Environ Health Perspect. 106 Suppl 1 (1998) 289-295. 
[43] W. Droge, Free radicals in the physiological control of cell function, Physiol Rev. 82 (2002) 47-95.

[44] G.J. Hannon, P. Sun, A. Carnero, L.Y. Xie, R. Maestro, D.S. Conklin, D. Beach, MaRX: an approach to genetics in mammalian cells, Science. 283 (1999) 1129-1130.

[45] A. Carnero, J.D. Hudson, G.J. Hannon, D.H. Beach, Loss-of-function genetics in mammalian cells: the p53 tumor suppressor model, Nucleic Acids Res. 28 (2000) 2234-2241.

[46] M. Vergel, A. Carnero, Bypassing cellular senescence by genetic screening tools, Clin Transl Oncol. 12 (2010) 410-417.

[47] M.V. Guijarro, M.E. Castro, L. Romero, V. Moneo, A. Carnero, Large scale genetic screen identifies MAP17 as protein bypassing TNF-induced growth arrest, J Cell Biochem. 101 (2007) 112-121.

[48] T. Blasco, J. Aramayona, A. Alcalde, J. Catalan, M. Sarasa, V. Sorribas, Rat kidney MAP17 induces cotransport of Na-mannose and Na-glucose in Xenopus laevis oocytes., Am J Physiol Renal Physiol. 285 (2003) F799-810.

[49] C. Jaeger, B. Schaefer, R. Wallich, M. Kramer, The membrane-associated protein pKe\#192/MAP17 in human keratinocytes., J Invest Dermatol 2000. 115 (2000) 375-380.

[50] O. Kocher, P. Cheresh, L.F. Brown, S.W. Lee, Identification of a novel gene, selectively up-regulated in human carcinomas, using the differential display technique, Clin Cancer Res. 1 (1995) 1209-1215.

[51] O. Kocher, P. Cheresh, S.W. Lee, Identification and partial characterization of a novel membrane-associated protein (MAP17) up-regulated in human carcinomas and modulating cell replication and tumor growth, Am J Pathol. 149 (1996) 493-500.

[52] M.A. Lanaspa, H. Giral, S.Y. Breusegem, N. Halaihel, G. Baile, J. Catalan, J.A. Carrodeguas, N.P. Barry, M. Levi, V. Sorribas, Interaction of MAP17 with NHERF3/4 induces translocation of the renal Na/Pi lla transporter to the trans-Golgi, Am J Physiol Renal Physiol. 292 (2007) F230-242.

[53] E. Delabesse, S. Ogilvy, M.A. Chapman, S.G. Piltz, B. Gottgens, A.R. Green, Transcriptional regulation of the SCL locus: identification of an enhancer that targets the primitive erythroid lineage in vivo, Mol Cell Biol. 25 (2005) 5215-5225.

[54] B. Gottgens, L.M. Barton, M.A. Chapman, A.M. Sinclair, B. Knudsen, D. Grafham, J.G. Gilbert, J. Rogers, D.R. Bentley, A.R. Green, Transcriptional regulation of the stem cell leukemia gene (SCL)--comparative analysis of five vertebrate SCL loci, Genome Res. 12 (2002) 749-759.

[55] M. Noh, H. Yeo, J. Ko, H.K. Kim, C.H. Lee, MAP17 is associated with the T-helper cell cytokine-induced down-regulation of filaggrin transcription in human keratinocytes, Exp Dermatol. 19 (2010) 355-362.

[56] M.V. Guijarro, J.F. Leal, J. Fominaya, C. Blanco-Aparicio, S. Alonso, M. Lleonart, J. Castellvi, L. Ruiz, Y.C.S. Ramon, A. Carnero, MAP17 overexpression is a common characteristic of carcinomas, Carcinogenesis. 28 (2007) 1646-1652.

[57] M.V. Guijarro, M. Vergel, J.J. Marin, S. Munoz-Galvan, I. Ferrer, S.R. Cajal, G. Roncador, C. Blanco-Aparicio, A. Carnero, p38alpha limits the contribution of MAP17 to cancer progression in breast tumors, Oncogene. (2012).

[58] B. Vogelstein, K.W. Kinzler, Cancer genes and the pathways they control, Nat Med. 10 (2004) 789-799.

[59] M.V. Guijarro, J.F. Leal, C. Blanco-Aparicio, S. Alonso, J. Fominaya, M. Lleonart, J. Castellvi, S. Ramon y Cajal, A. Carnero, MAP17 enhances the malignant behavior of tumor cells through ROS increase, Carcinogenesis. 28 (2007) 20962104.

[60] T. Blasco, J.J. Aramayona, A.I. Alcalde, J. Catalan, M. Sarasa, V. Sorribas, Rat kidney MAP17 induces cotransport of Na-mannose and Na-glucose in Xenopus laevis oocytes, Am J Physiol Renal Physiol. 285 (2003) F799-810.

[61] M. Veyhl, J. Spangenberg, B. Puschel, R. Poppe, C. Dekel, G. Fritzsch, W. Haase, H. Koepsell, Cloning of a membrane-associated protein which modifies activity 
and properties of the $\mathrm{Na}(+)$-D-glucose cotransporter, J Biol Chem. 268 (1993) 25041-25053.

[62] O. Kocher, N. Comella, K. Tognazzi, L.F. Brown, Identification and partial characterization of PDZK 1: a novel protein containing PDZ interaction domains, Lab Invest. 78 (1998) 117-125.

[63] D.L. Silver, N. Wang, S. Vogel, Identification of small PDZK1-associated protein, DD96/MAP17, as a regulator of PDZK1 and plasma high density lipoprotein levels, J Biol Chem. 278 (2003) 28528-28532.

[64] S. Pribanic, S. Gisler, D. Bacic, C. Madjdpour, N. Hernando, V. Sorribas, A. Gantenbein, J. Biber, H. Murer, Interactions of MAP17 with the NaPi-lla/PDZK 1 protein complex in renal proximal tubular cells. . Am J Physiol Renal Physiol. 285 (2003) F784-791.

[65] R. Cunningham, R. Biswas, D. Steplock, S. Shenolikar, E. Weinman, Role of NHERF and scaffolding proteins in proximal tubule transport, Urol Res. 38 (2010) 257-262.

[66] A. Claperon, M. Mergey, L. Fouassier, Roles of the scaffolding proteins NHERF in liver biology, Clin Res Hepatol Gastroenterol. 35 (2011) 176-181.

[67] N.C. Zachos, C. Hodson, O. Kovbasnjuk, X. Li, W.R. Thelin, B. Cha, S. Milgram, M. Donowitz, Elevated intracellular calcium stimulates NHE3 activity by an IKEPP (NHERF4) dependent mechanism, Cell Physiol Biochem. 22 (2008) 693-704.

[68] A. Claperon, M. Mergey, L. Fouassier, Roles of the scaffolding proteins NHERF in liver biology, Gastroenterol Clin Biol. (2011).

[69] R. Villa-Bellosta, M. Barac-Nieto, S.Y. Breusegem, N.P. Barry, M. Levi, V. Sorribas, Interactions of the growth-related, type Ilc renal sodium/phosphate cotransporter with PDZ proteins, Kidney Int. 73 (2008) 456-464.

[70] D. Reczek, M. Berryman, A. Bretscher, Identification of EBP50: A PDZ-containing phosphoprotein that associates with members of the ezrin-radixin-moesin family, J Cell Biol. 139 (1997) 169-179.

[71] J. Ingraffea, D. Reczek, A. Bretscher, Distinct cell type-specific expression of scaffolding proteins EBP50 and E3KARP: EBP50 is generally expressed with ezrin in specific epithelia, whereas E3KARP is not, Eur J Cell Biol. 81 (2002) 61-68.

[72] S. Shenolikar, J.W. Voltz, R. Cunningham, E.J. Weinman, Regulation of ion transport by the NHERF family of PDZ proteins, Physiology (Bethesda). 19 (2004) 362-369.

[73] G. Lamprecht, U. Seidler, The emerging role of PDZ adapter proteins for regulation of intestinal ion transport, Am J Physiol Gastrointest Liver Physiol. 291 (2006) G766777.

[74] A. Cinar, M. Chen, B. Riederer, O. Bachmann, M. Wiemann, M. Manns, O. Kocher, U. Seidler, NHE3 inhibition by CAMP and Ca2+ is abolished in PDZ-domain protein PDZK 1-deficient murine enterocytes, J Physiol. 581 (2007) 1235-1246.

[75] T. Sugiura, Y. Kato, T. Wakayama, D.L. Silver, Y. Kubo, S. Iseki, A. Tsuji, PDZK 1 regulates two intestinal solute carriers (Slc15al and Slc22a5) in mice, Drug Metab Dispos. 36 (2008) 1181-1188.

[76] J. Hillesheim, B. Riederer, B. Tuo, M. Chen, M. Manns, J. Biber, C. Yun, O. Kocher, U. Seidler, Down regulation of small intestinal ion transport in PDZK 1(CAP70/NHERF3) deficient mice, Pflugers Arch. 454 (2007) 575-586.

[77] S.M. Gisler, S. Pribanic, D. Bacic, P. Forrer, A. Gantenbein, L.A. Sabourin, A. Tsuji, Z.S. Zhao, E. Manser, J. Biber et al, PDZK 1: I. a major scaffolder in brush borders of proximal tubular cells, Kidney Int. 64 (2003) 1733-1745.

[78] M. Gentzsch, L. Cui, A. Mengos, X.B. Chang, J.H. Chen, J.R. Riordan, The PDZbinding chloride channel CIC-3B localizes to the Golgi and associates with cystic fibrosis transmembrane conductance regulator-interacting PDZ proteins, J Biol Chem. 278 (2003) 6440-6449.

[79] J. Inove, T. Otsuki, A. Hirasawa, I. Imoto, Y. Matsuo, S. Shimizu, M. Taniwaki, J. Inazawa, Overexpression of PDZK1 within the 1q12-q22 amplicon is likely to be associated with drug-resistance phenotype in multiple myeloma, Am J Pathol. 165 (2004) 71-81.

[80] E.M. Wright, E. Turk, The sodium/glucose cotransport family SLC5, Pflugers Arch. 447 (2004) 510-518. 
[81] A.M. Pajor, B.A. Hirayama, E.M. Wright, Molecular evidence for two renal $\mathrm{Na}+$ /glucose cotransporters, Biochim Biophys Acta. 1106 (1992) 216-220.

[82] A.M. Pajor, E.M. Wright, Cloning and functional expression of a mammalian $\mathrm{Na}+$ /nucleoside cotransporter. A member of the SGLT family, J Biol Chem. 267 (1992) 3557-3560.

[83] E.M. Wright, D.D. Loo, M. Panayotova-Heiermann, M.P. Lostao, B.H. Hirayama, B. Mackenzie, K. Boorer, G. Zampighi, 'Active' sugar transport in eukaryotes, J Exp Biol. 196 (1994) 197-212.

[84] C.Y. Huang, J.K. Hsiao, Y.Z. Lu, T.C. Lee, L.C. Yu, Anti-apoptotic PI3K/AKt signaling by sodium/glucose transporter 1 reduces epithelial barrier damage and bacterial translocation in intestinal ischemia, Lab Invest. 91 (2011) 294-309.

[85] Y. Hanabata, Y. Nakajima, K.I. Morita, K. Kayamori, K. Omura, Coexpression of SGLT1 and EGFR is associated with tumor differentiation in oral squamous cell carcinoma, Odontology. (2011).

[86] Z. Weihua, R. Tsan, W.C. Huang, Q. Wu, C.H. Chiu, I.J. Fidler, M.C. Hung, Survival of cancer cells is maintained by EGFR independent of its kinase activity, Cancer Cell. 13 (2008) 385-393.

[87] M. Ristow, Oxidative metabolism in cancer growth, Curr Opin Clin Nutr Metab Care. 9 (2006) 339-345.

[88] R.J. Shaw, Glucose metabolism and cancer, Curr Opin Cell Biol. 18 (2006) 598-608.

[89] J.W. Kim, C.V. Dang, Cancer's molecular sweet tooth and the Warburg effect, Cancer Res. 66 (2006) 8927-8930.

[90] J.W. Kim, I. Tchernyshyov, G.L. Semenza, C.V. Dang, HIF-1-mediated expression of pyruvate dehydrogenase kinase: a metabolic switch required for cellular adaptation to hypoxia, Cell Metab. 3 (2006) 177-185.

[91] V. Ganapathy, M. Thangaraju, P.D. Prasad, Nutrient transporters in cancer: relevance to Warburg hypothesis and beyond, Pharmacol Ther. 121 (2009) 2940.

[92] O. Warburg, The metabolism of tumors, London: Constable. (1930).

[93] O. Warburg, On the origin of cancer cells, Science. 123 (1956) 309-314.

[94] D.C. Wallace, Mitochondria and cancer: Warburg addressed, Cold Spring Harb Symp Quant Biol. 70 (2005) 363-374.

[95] M. Brandon, P. Baldi, D.C. Wallace, Mitochondrial mutations in cancer, Oncogene. 25 (2006) 4647-4662.

[96] J.A. Engelman, L.C. Cantley, A sweet new role for EGFR in cancer, Cancer Cell. 13 (2008) 375-376.

[97] D.A. Eberhard, G. Giaccone, B.E. Johnson, Biomarkers of response to epidermal growth factor receptor inhibitors in Non-Small-Cell Lung Cancer Working Group: standardization for use in the clinical trial setting, J Clin Oncol. 26 (2008) 983-994.

[98] M. Thangaraju, E. Gopal, P.M. Martin, S. Ananth, S.B. Smith, P.D. Prasad, E. Sterneck, V. Ganapathy, SLC5A8 triggers tumor cell apoptosis through pyruvatedependent inhibition of histone deacetylases, Cancer Res. 66 (2006) 11560 11564.

[99] M. Thangaraju, S.K. Karunakaran, S. Itagaki, E. Gopal, S. Elangovan, P.D. Prasad, V. Ganapathy, Transport by SLC5A8 with subsequent inhibition of histone deacetylase 1 (HDACl) and HDAC3 underlies the antitumor activity of 3bromopyruvate, Cancer. 115 (2009) 4655-4666.

[100] J. Pouyssegur, F. Mechta-Grigoriou, Redox regulation of the hypoxia-inducible factor, Biol Chem. 387 (2006) 1337-1346.

[101] J. Chiche, K. IIC, M.C. Brahimi-Horn, J. Pouyssegur, Membrane-bound carbonic anhydrases are key $\mathrm{pH}$ regulators controlling tumor growth and cell migration, Adv Enzyme Regul. 50 (2010) 20-33.

[102] M.C. Brahimi-Horn, J. Pouyssegur, Hypoxia in cancer cell metabolism and pH regulation, Essays Biochem. 43 (2007) 165-178.

[103] R.A. Cardone, V. Casavola, S.J. Reshkin, The role of disturbed pH dynamics and the $\mathrm{Na}+\mathrm{H}+$ exchanger in metastasis, Nat Rev Cancer. 5 (2005) 786-795. 
[104] L. Counillon, J. Pouyssegur, The expanding family of eucaryotic $\mathrm{Na}(+) / H(+)$ exchangers, J Biol Chem. 275 (2000) 1-4.

[105] C. Sardet, A. Franchi, J. Pouyssegur, Molecular cloning, primary structure, and expression of the human growth factor-activatable $\mathrm{Na} / / \mathrm{H}+$ antiporter, Cell. 56 (1989) 271-280.

[106] M.S. Ullah, A.J. Davies, A.P. Halestrap, The plasma membrane lactate transporter MCT4, but not MCT1, is up-regulated by hypoxia through a HIF-lalphadependent mechanism, J Biol Chem. 281 (2006) 9030-9037.

[107] S.A. Karumanchi, L. Jiang, B. Knebelmann, A.K. Stuart-Tilley, S.L. Alper, V.P. Sukhatme, VHL tumor suppressor regulates $\mathrm{Cl} / \mathrm{HCO}$ - exchange and $\mathrm{Na}+/ \mathrm{H}+$ exchange activities in renal carcinoma cells, Physiol Genomics. 5 (2001) 119-128.

[108] S.K. Parks, J. Chiche, J. Pouyssegur, pH control mechanisms of tumor survival and growth, J Cell Physiol. 226 (2011) 299-308.

[109] P. Storz, Reactive oxygen species in tumor progression, Front Biosci. 10 (2005) 18811896.

[1 10] M. Marra, I.M. Sordelli, A. Lombardi, M. Lamberti, L. Tarantino, A. Giudice, P. Stiuso, A. Abbruzzese, R. Sperlongano, M. Accardo et al, Molecular targets and oxidative stress biomarkers in hepatocellular carcinoma: an overview, J Transl Med. 9 (2011) 171.

[1111] J.P. Fruehauf, F.L. Meyskens, Jr., Reactive oxygen species: a breath of life or death?, Clin Cancer Res. 13 (2007) 789-794.

[112] S. Simizu, M. Takada, K. Umezawa, M. Imoto, Requirement of caspase-3(-like) protease-mediated hydrogen peroxide production for apoptosis induced by various anticancer drugs, J Biol Chem. 273 (1998) 26900-26907.

[113] T. Yoshikawa, S. Kokura, K. Tainaka, Y. Naito, M. Kondo, A novel cancer therapy based on oxygen radicals, Cancer Res. 55 (1995) 1617-1620.

[1 14] C.F. Nathan, B.A. Arrick, H.W. Murray, N.M. DeSantis, Z.A. Cohn, Tumor cell antioxidant defenses. Inhibition of the glutathione redox cycle enhances macrophage-mediated cytolysis, J Exp Med. 153 (1981) 766-782.

[115] C.F. Nathan, Z.A. Cohn, Antitumor effects of hydrogen peroxide in vivo, J Exp Med. 154 (1981) 1539-1553.

[116] M. Sanchez-Carbayo, N.D. Socci, J. Lozano, F. Saint, C. Cordon-Cardo, Defining molecular profiles of poor outcome in patients with invasive bladder cancer using oligonucleotide microarrays, J Clin Oncol. 24 (2006) 778-789.

[117] G. Finak, N. Bertos, F. Pepin, S. Sadekova, M. Souleimanova, H. Zhao, H. Chen, G. Omeroglu, S. Meterissian, A. Omeroglu et al, Stromal gene expression predicts clinical outcome in breast cancer, Nat Med. 14 (2008) 518-527.

[118] H. Pei, L. Li, B.L. Fridley, G.D. Jenkins, K.R. Kalari, W. Lingle, G. Petersen, Z. Lou, L. Wang, FKBP51 affects cancer cell response to chemotherapy by negatively regulating Akt, Cancer Cell. 16 (2009) 259-266.

[119] D.H. Ki, H.C. Jeung, C.H. Park, S.H. Kang, G.Y. Lee, W.S. Lee, N.K. Kim, H.C. Chung, S.Y. Rha, Whole genome analysis for liver metastasis gene signatures in colorectal cancer, Int J Cancer. 121 (2007) 2005-2012.

[120] N.D. Hendrix, R. WU, R. Kuick, D.R. Schwartz, E.R. Fearon, K.R. Cho, Fibroblast growth factor 9 has oncogenic activity and is a downstream target of Wnt signaling in ovarian endometrioid adenocarcinomas, Cancer Res. 66 (2006) 1354-1362. 


\section{Figure legend}

Figure 1: Representative pictures of human tumors overexpressing MAP17. A) Colorectal carcinoma. B) Undifferentiated cervical carcinoma. C) Mammary carcinoma. D) Lung adenocarcinoma. Pictures show tumor cells, characterized by nuclear displasia, heavily stained for MAP17 expression. Non tumoral cells forming the stroma, show no staining.

Figure 2: Overexpression of MAP17 in human tumors. Graphs show overexpression of MAP17 mRNA in tumors compared with non-tumoral tissue in a large-scale microarray data set from different studies (www.oncomine.com). A) From [116]. B) From [117]. C) From [1 18]. D) From [119]. E) From [120]. F) From [120].

Figure 3: A) Scheme of the possible functional role of MAP17 as an anchor for PDZK1-membrane transporter complexes. B) Scheme of hypothetic functional relationship between SGLT1 and MAP17 through NHERF. SGLT1 increase in tumor cells to respond to high glucose demand, while MAP17 increase to regulate the subsequent intracellular acidification through NHeRF binding and localizing $\mathrm{pH}$ detoxifying enzymes in the membrane. 


\section{Abbreviations}

PDZ, Post-synaptic density 95, Disc large, Zonula occludens 1 PDZK 1, PDZ domain-containing 1

CLAMP, c-terminal linking and modulating protein;

CAP70, CFTR-associated protein, 70-KD

MAP17, Membrane associated protein 17 KD

$\mathrm{NHeRF}, \mathrm{Na}+\mathrm{H}+$ exchanger regulatory factor

IKEPP, Intestinal and kidney enriched PDZ protein

SGLT, Na+/glucose cotransporter 